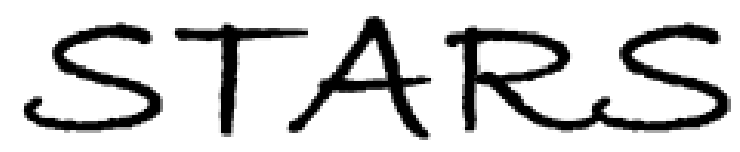

University of Central Florida

STARS

$1-1-2010$

\title{
The 1998 November 14 Occultation of Gsc 0622-00345 By Saturn. I. Techniques for Ground-Based Stellar Occultations
}

Joseph Harrington

University of Central Florida

Richard G. French

Find similar works at: https://stars.library.ucf.edu/facultybib2010 University of Central Florida Libraries http://library.ucf.edu

This Article is brought to you for free and open access by the Faculty Bibliography at STARS. It has been accepted for inclusion in Faculty Bibliography 2010 s by an authorized administrator of STARS. For more information, please contact STARS@ucf.edu.

\section{Recommended Citation}

Harrington, Joseph and French, Richard G., "The 1998 November 14 Occultation of Gsc 0622-00345 By Saturn. I. Techniques for Ground-Based Stellar Occultations" (2010). Faculty Bibliography 2010s. 229. https://stars.library.ucf.edu/facultybib2010/229

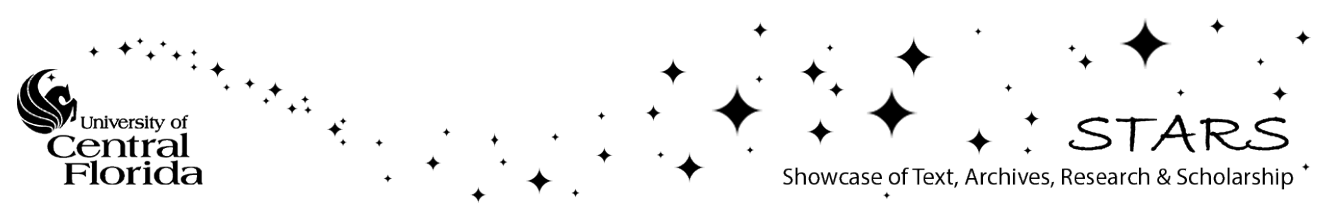




\title{
THE 1998 NOVEMBER 14 OCCULTATION OF GSC 0622-00345 BY SATURN. I. TECHNIQUES FOR GROUND-BASED STELLAR OCCULTATIONS
}

\author{
Joseph HARrington ${ }^{1,3}$ AND RiCHARD G. FRENCH ${ }^{2,3}$ \\ ${ }^{1}$ Planetary Sciences Group, Department of Physics, University of Central Florida, Orlando, FL 32816-2385, USA; jh@physics.ucf.edu \\ ${ }^{2}$ Astronomy Department, Wellesley College, Wellesley, MA 02481, USA; rfrench@wellesley.edu \\ Received 2009 July 23; accepted 2009 December 6; published 2010 May 19
}

\begin{abstract}
On 1998 November 14, Saturn and its rings occulted the star GSC 0622-00345. We observed atmospheric immersion with NSFCAM at the National Aeronautics and Space Administration's Infrared Telescope Facility on Mauna Kea, Hawaii. Immersion occurred at 55.5 S planetocentric latitude. A $2.3 \mu \mathrm{m}, \mathrm{CH}_{4}$-band filter suppressed reflected sunlight. Atmospheric emersion and ring data were not successfully obtained. We describe our observation, light curve production, and timing techniques, including improvements in aperture positioning, removal of telluric scintillation effects, and timing. Many of these techniques are known within the occultation community, but have not been described in the reviewed literature. We present a light curve whose signal-to-noise ratio per scale height is 267 , among the best ground-based signals yet achieved, despite a disadvantage of up to 8 mag in the stellar flux compared to prior work.
\end{abstract}

Key words: atmospheric effects - infrared: planetary systems - occultations - planets and satellites: individual (Saturn) - techniques: image processing - techniques: photometric

Online-only material: tar.gz

\section{INTRODUCTION}

Stellar occultations by planets offer among the highest spatial resolution of any astronomical observing technique, competing even with atmospheric descent probes for certain measurements. From Earth, spatial resolution is limited by the projected stellar beam size and Fresnel diffraction, the latter giving $\sim 1 \mathrm{~km}$ resolution for solar system giant planets for visible and near-IR wavelengths. This is sufficient to reveal fine structure in a planetary ring or propagating gravity (buoyancy) waves in an atmosphere. However, the occultation technique is demanding, and failing to optimize even a single parameter of an observation can significantly reduce the quality of the derived light curve. Occultation observers have thus developed numerous specialized techniques to improve their data. Many of these have never been described in the literature.

On 1998 November 14, Saturn and its rings occulted GSC 0622-00345, as predicted by Bosh \& McDonald (1992). We obtained a light curve for atmospheric immersion, based on infrared imaging observations at the NASA Infrared Telescope Facility (IRTF) on Mauna Kea, HI. Figure 1 shows our viewing circumstances and Table 1 gives parameters of the event.

Occultation cadences are typically from a few to $10 \mathrm{~Hz}$, and in the case of lunar occultations they can be much higher. These rates are sufficient for the temporal resolution of flux variations caused by scintillation (variable refractive focusing and defocusing of the stellar beam) in Earth's atmosphere. This is typically the limiting noise source for occultations by bright stars. During our observations, we monitored Rhea, which experienced approximately the same scintillation effects as the star, just arcseconds away. We were thus able to use measurements of Rhea's (assumed constant) flux to compensate

\footnotetext{
3 Visiting Astronomer at the Infrared Telescope Facility, which is operated by the University of Hawaii under Cooperative Agreement No. NCC 5-538 with the National Aeronautics and Space Administration, Science Mission Directorate, Planetary Astronomy Program.
}

for scintillation. This, in combination with other techniques, produced a light curve of very high quality.

Here, we present a full accounting of the methods we employed to observe the event and derive the light curve, including those previously undocumented. Section 2 describes observing techniques, data reduction methods, and the light curve. It also discusses an attempt to apply optimal photometry to light curve production and thoughts on improving instrument interfaces to avoid failures such as our lost egress observation. Section 3 presents a method for independently verifying the system timing solution. Section 4 offers brief conclusions. We include the light curve and software for analyzing occultation timing data as electronic supplements. Paper II (Harrington et al. 2010) uses the derived light curve to investigate the atmosphere of Saturn.

\section{OBSERVATIONS AND LIGHT CURVE}

The NSFCAM InSb array camera (Shure et al. 1994) recorded the event in "movieburst" mode. This mode writes a time sequence of images to computer memory and then saves the set to disk after the observations finish. We recorded three subarrays derived from the full $256 \times 256$-pixel array. These boxes contained the star $(28 \times 32$ pixels $)$, Rhea $(28 \times 32$ pixels $)$, and a piece of blank sky $(16 \times 16$ pixels $)$, placed as shown in Figure 1 . The larger boxes were originally $32 \times 32$ pixels, but a camera software error overwrote four edge columns. No critical data were lost and the error is now fixed. Table 2 gives additional observation and light curve parameters.

The limit to spatial resolution in a stellar occultation is generally the larger of the Fresnel scale and the projected size of the occulted star. For typical in situ spacecraft occultations, both are very small compared to the sky-plane distance between samples, but for Earth-based work either can dominate. In the case of $28 \mathrm{Sgr}$, the projected stellar diameter at the distance of Saturn was $\sim 20 \mathrm{~km}$, much larger than the $\sim 1 \mathrm{~km}$ Fresnel diffraction scale, resulting in significant smoothing of the 
Table 1

Occultation Parameters

\begin{tabular}{|c|c|c|}
\hline Description & Value & Comment \\
\hline Star & GSC 0622-00345 & \\
\hline R.A. & $1 \mathrm{~h} 49 \mathrm{~m} 54^{\prime \prime} .358$ & $\mathrm{~J} 2000^{\mathrm{a}}$ \\
\hline Decl. & $8^{\circ} 23^{\prime} 12^{\prime \prime} .56$ & $\mathrm{~J} 2000^{\mathrm{a}}$ \\
\hline$V$ magnitude & 11.002 & $\mathrm{a}$ \\
\hline$K$ magnitude & 8.395 & $\mathrm{~b}$ \\
\hline Spectral Type & K5 & \\
\hline Proj. diam. at Saturn & $\sim 0.7 \mathrm{~km}$ & \\
\hline Half-light latitude & $55.5 \mathrm{~S}$ & Planetocentric \\
\hline Fresnel scale & $1.2 \mathrm{~km}$ & $\sqrt{\lambda d / 2}$ \\
\hline Sky-plane velocity & $17.940 \mathrm{~km} \mathrm{~s}^{-1}$ & \\
\hline Perpendicular velocity, $v_{\perp}$ & $12.802 \mathrm{~km} \mathrm{~s}^{-1}$ & vs. oblate limb \\
\hline Ring opening angle & -14.6772 & \\
\hline Pole position angle & 1.5134 & $\mathrm{~J} 2000$ \\
\hline Saturn's geocent. dist., $d$ & $1.25168 \times 10^{9} \mathrm{~km}$ & \\
\hline
\end{tabular}

Notes.

a Tycho-2 record 131066, Høg et al. (2000), $\sigma_{\text {pos }} \sim 65 \mathrm{mas}, V=V T-$ $0.090(B T-V T)$, using catalog $V T$ and $B T$ magnitudes.

b 2MASS 01495435+0823123, Skrutskie et al. (2006).

ring and atmospheric occultation profiles (e.g., Harrington et al. 1993, Figure 4). To estimate the angular diameter of GSC 0622-00345, we first used the color index $V-K=2.607$ (Table 1) to infer a dwarf spectral type of K5 (Glass 1999). The stellar diameter for this spectral class (Lang 1992) yields an estimated angular diameter of 0.12 mas, or $0.7 \pm 0.2 \mathrm{~km}$ at Saturn's distance, where the error bar takes into account the possible effects of modest interstellar reddening. Even with a large uncertainty, the projected size of the occulted star is less than the Fresnel scale, which thus determines the intrinsic spatial resolution of the occultation.

The $2.3 \mu \mathrm{m}$ filter is centered on a methane band, where Saturn's atmosphere strongly absorbs sunlight, so contrast between the star and Saturn is very high. The rings are bright at this wavelength, but their spatial separation from the immersion latitude made it practical to subtract their scattered light (see below).

At the half-light time (see below), seeing was 0.9 FWHM and the star was at 1.3 air masses and setting. The night was clear, but Rhea's flux varied by up to $8 \%$ in successive frames $(2.3 \% \mathrm{rms})$ due to scintillation. Rhea was nearly as bright as the unocculted star (see Table 2) and had a point-spread function (PSF) that very closely matched that of the star in each frame. We thus used Rhea as a standard for flux and position and attempted to use it as a PSF standard. In contrast to the scintillation, sky emission was very steady and low, based on the separately recorded sky box.

To obtain sub-pixel accuracy in image shifts, photometry aperture placement, and scattered-light subtraction, we subsampled by a factor of 10 using nearest-neighbor sampling (a factor of 5 gave essentially identical results). We performed all operations on the expanded images, except as noted below. These subpixels allowed a reasonable approximation to a circular photometry aperture that included partial pixels.

After debiasing, flat-fielding, and interpolating three hot pixels that were not near the star or Rhea, we centroided Rhea in all images. The relative position of the star in two well-separated sets of 100 pre-occultation frames determined the Rhea-star offset and the offset's drift with time. This allowed accurate placement of the Saturn and ring background template (described below) and photometry apertures, even when the star was fully occulted. A third set of stellar centroids confirmed that the pre-
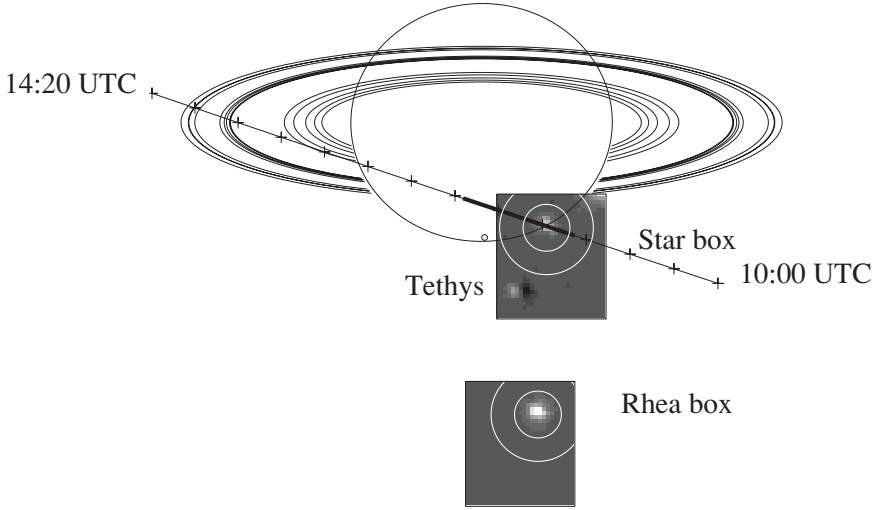

Rhea box

Sky box

Figure 1. Occultation geometry, subarray placement, and photometry apertures at the half-light time. The light line marks the path of GSC 0622-00345 relative to Saturn, with crosses every 20 minutes. The heavier portion gives the span of the data set. The relative positions of the star, Rhea, and sky subarrays were constant. Telescope tracking kept the star in roughly the same pixel while Saturn and its moons drifted through. Tethys's motion relative to Saturn is evident by the position mismatch between the half-light data and the scattered-light template. We computed the template from post-occultation frames and subtracted it from the data to produce the star frame shown here.

dicted stellar positions were accurate to within 0.'045 (rms), or 0.15 unscaled pixels. To estimate Saturn's position (for template placement, see below), we added the Rhea-Saturn relative skyplane offset to Rhea's measured position in each frame. The Jet Propulsion Laboratory's Horizons On-Line Ephemeris System Web site ${ }^{4}$ generated the relevant positions on 2004 January 9 based on the DE-0406, LE-0406, and SAT136 ephemerides. Our derived Rhea-star drift rate closely matched the ephemeris prediction.

The small amount of scattered light from Saturn and the rings complicated stellar photometry by requiring the subtraction of a scattered-light template. We made a template by calculating the shifted, masked average (see below) of a set of post-immersion images, each divided by Rhea's flux in that frame and shifted to align the predicted position of Saturn in all frames. All shifts both in creation and application of the template were in units of integer subpixels, so no interpolation occurred.

A shifted, masked average starts with two similar 3D "stacks," respectively containing the images and their bad pixel masks (in our case, all the masks are the same). In a mask, pixel values are one for good pixels and zero for bad pixels. The technique proceeds as follows. Pad the stacks with zeros to prevent good data from shifting outside the boundaries. Multiply the image stack by the mask stack to set bad pixels to zero, shift the images to align them, and apply the same shifts to the masks. Collapse each stack into a 2D image by summing along the image index dimension. At each pixel location, divide the image sum by the mask sum to calculate the shifted mask average. Each stacked image shifted differently, so the bad pixels shifted out of alignment with one another. The result has no bad pixels and was calculated without interpolation. The mask sum gives the number of good pixels contributing to the average at each pixel.

To apply the template, we shifted it according to Saturn's predicted position (see above) in each occultation frame, multiplied it by Rhea's flux in that frame, down-sampled to the

\footnotetext{
4 http://ssd.jpl.nasa.gov/?horizons
} 
Table 2

Observation and Light-curve Parameters

\begin{tabular}{|c|c|c|}
\hline Description & Value & Comment \\
\hline Telescope & IRTF & \\
\hline Instrument & NSFCAM & \\
\hline Image scale & $0 ! 30$ pixel $^{-1}$ & \\
\hline Wavelength, $\lambda$ & $2.28 \mu \mathrm{m}$ & "Spencer 2.3" filter \\
\hline Bandpass, FWHM & $0.17 \mu \mathrm{m}$ & \\
\hline Time between frame starts & $0.2128 \mathrm{~s}$ & \\
\hline Exposure time & $0.1276 \mathrm{~s}$ & Dead time of $0.0852 \mathrm{~s}$, see the text \\
\hline Readout time & $0.009469 \mathrm{~s}$ & \\
\hline Non-destructive reads (NDR) & 8 & \\
\hline Gain & $10 \mathrm{e}^{-} \mathrm{DN}^{-1}$ & \\
\hline Read noise & $19 \mathrm{e}^{-}$ & $55 \mathrm{e}^{-} \mathrm{read}^{-1} / \sqrt{\mathrm{NDR}}$ \\
\hline \multicolumn{3}{|l|}{ Frame mid-times: } \\
\hline First frame & 11:05:02.622 UTC & \\
\hline First frame in upper baseline average & 11:16:44.939 UTC & \\
\hline First frame in lower baseline average & 11:26:19.562 UTC & No evidence of star in frames \\
\hline Last frame & 11:56:07.065 UTC & \\
\hline Number of frames & 14,400 & \\
\hline Number of frames per baseline average & 300 & \\
\hline \multicolumn{3}{|l|}{ Photometry (upper baseline interval): } \\
\hline Star full flux & $5439 \mathrm{DN}$ & Above lower baseline \\
\hline Rhea full flux & $5216 \mathrm{DN}$ & Above lower baseline \\
\hline Sky box & $12.55 \mathrm{DN} \mathrm{pixel}^{-1}$ & \\
\hline Variability (upper baseline interval): & & Standard deviation \\
\hline Star & $2.2 \%$ & Scintillation uncorrected \\
\hline Star & $1.5 \%$ & Scintillation corrected \\
\hline Sky box & $3.8 \%$ & \\
\hline $\mathrm{S} / \mathrm{N}$ per frame & 66 & \\
\hline $\mathrm{S} / \mathrm{N}$ per scale height & 267 & \\
\hline
\end{tabular}

original spatial resolution, subtracted from the calibrated stellar frame, up-sampled the result to the scaled resolution using nearest-neighbor sampling, and performed aperture photometry using the predicted stellar position.

The 0.15-pixel centering uncertainty would lead to slight misalignments of the template. In the region of the limb, the template's gradient is typically $0.0018 \mathrm{DN}$ per subpixel (the peak is $0.0054 \mathrm{DN}$ per subpixel). Both these values are found after multiplying by Rhea's mean flux from Table 2 and dividing by the square of the scaling (100, so that photometry yields an unscaled DN value like those in Table 2). The aperture boundary touches the limb gradient in only two places, however; so template shift errors contribute minimal noise. Shifting the template by one or two subpixels horizontally or vertically, subtracting it from the unshifted template, and performing aperture photometry yields -1.26 and $-2.10 \mathrm{DN}$ for the horizontal shifts and 1.21 and $2.08 \mathrm{DN}$ for the vertical shifts, respectively. The photon noise from the unocculted star is $23 \mathrm{DN}$, from the sky in the aperture is $12 \mathrm{DN}$, and from other sources is $15 \mathrm{DN}$ based on aperture photometry of the template. Template misalignment is thus a minor noise source even compared to photon noise.

The template-subtracted stellar frames were quite flat except in the corner that included some ring light (see Figure 1). Pixels in this region were in the sky annulus but not in the photometry aperture, and were efficiently rejected by taking the median of all good pixels in the sky annulus. Sky photometry for Rhea also used the median, for consistency, as did that in the preceding paragraph.

The stellar and Rhea photometry apertures both had diameters of 3". 6 , four times the seeing FWHM. The abutting sky annuli had outer diameters of 7".2 (see Figure 1). The photometry for the unocculted star and for Rhea closely followed each other, indicating that the main noise contributor was scintillation. We thus divided the stellar flux by Rhea's flux in each frame (just as we adjusted the template's flux).

The light curve's upper and lower baseline levels are the medians of sections of the data before and after immersion (see Table 2). We used the median to reduce the effect of nonGaussian residual scintillation. The lower baseline estimate and the template come from the same frames. Verifying that there is no star in the lower-baseline frames is crucial to the fits and inversions that follow. The lower baseline frames were aligned at the nominal Saturn position and added to make the template. Due to increasing ray bending, the nominal stellar position is a relatively stationary point on the limb at this time in the occultation. The template shows no evidence of the star and is smooth (see above). After template subtraction and scintillation correction, the median integrated value for the star aperture in the lower-baseline frames was $(1.5 \pm 6.0)^{-4}$ units of the full stellar flux over the level in the sky annulus. The mean was less than twice this value, and the uncertainty is the standard deviation of the mean. The star is effectively gone.

Figure 2 presents the light curve. Its per-frame signal-to-noise ratio $(\mathrm{S} / \mathrm{N})$ is 66 for the unocculted star. The French et al. (1978) atmospheric occultation noise parameter $\epsilon_{\phi}=\epsilon \sqrt{v_{\perp} / H}=$ 0.00375 , where $\epsilon$ is the standard deviation of the unocculted stellar flux in one-second bins divided by its mean, $v_{\perp}$ is given in Table 1 , and $H$, the scale height, is given in Table 1 of Paper II (baselines fixed case). The reciprocal of $\epsilon_{\phi}$ is the $S / N$ per scale height, and both are commonly used as figures of merit for occultation light curves. Our value of 267 exceeds even that of the extremely bright $28 \mathrm{Sgr}$ occultation. For example, the IRTF 28 Sgr immersion $\mathrm{S} / \mathrm{N}$ per scale height was 192 , 


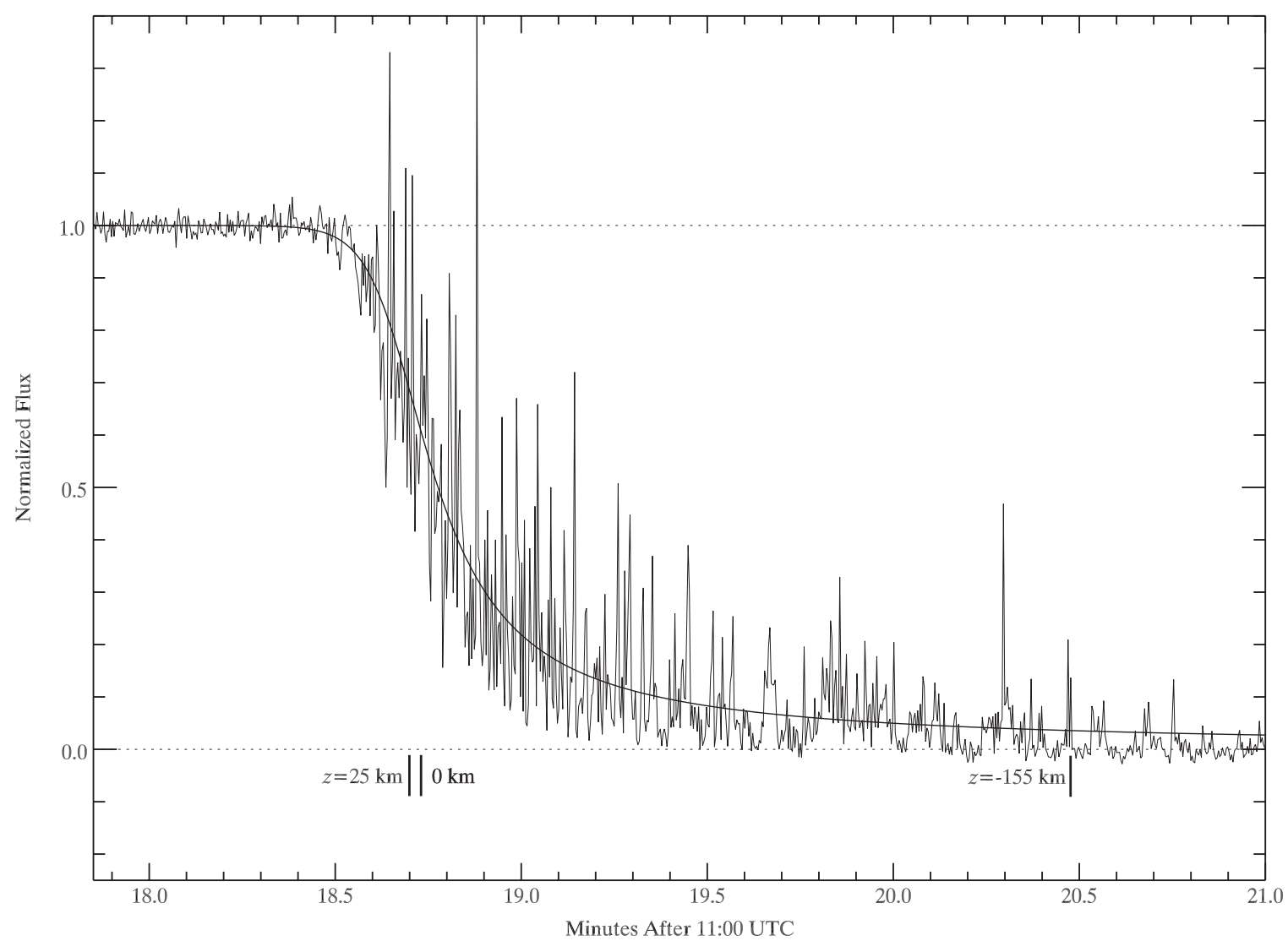

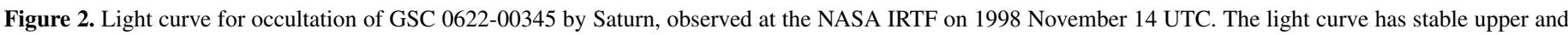

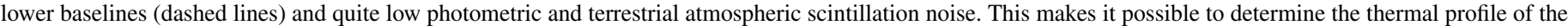

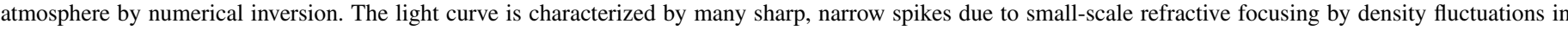

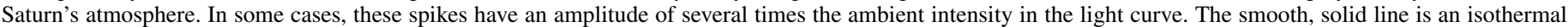

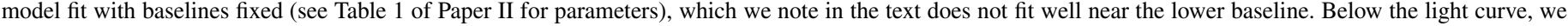
mark the location of the half-light point $(z=0 \mathrm{~km})$ and the extrema of the reliable region of the inversion, using altitudes determined by the inversion.

using the fractional flux standard deviation per frame of $1.7 \%$ and exposure time of $0.25 \mathrm{~s}$ reported by Harrington et al. (1993) and $H / v_{\perp}=2.66$ reported by Hubbard et al. (1997). The raw $\mathrm{S} / \mathrm{N}$ for these occultations is limited by scintillation rather than by photon statistics, eliminating 28 Sgr's 8 mag brightness advantage over the event reported here. Our slightly slower event, improvements in infrared array technology, and the analysis methods described herein account for the rest. The electronic supplement to this article includes the light curve data file.

A histogram of our upper baseline residuals closely follows a Gaussian distribution to $\pm 3 \sigma$, but at $>+3 \sigma$ it is slightly above the Gaussian, perhaps because scintillation produces occasional, transient flashes. The standard deviation in the difference between adjacent-frame fluxes is $>93 \%$ of that expected for uncorrelated errors and reaches $\sim 99 \%$ over an interval of $1 \mathrm{~s}$. The residual correlation may come from the $<5 \mathrm{~ms}$ interval between reads of the star and Rhea pixels. The power spectrum of the noise is thus still slightly stronger at lower wavenumbers than at higher wavenumbers (see below). In the occultation itself, one would expect correlated structure on the scale of the projected stellar diameter or the Fresnel diffraction scale (see Table 1), but both of these are below the $2.7 \mathrm{~km}$ sky-plane resolution of the light curve.

We computed the nominal occultation track from the ephemeris, and then offset by an additional $500 \mathrm{~km} \mathrm{E}$ and $500 \mathrm{~km}$ $\mathrm{N}$, relative to Saturn's center, so that the absolute radius of the half-light level matched the oblate half-light surface determined by Hubbard et al. (1997) from 28 Sgr occultation observations. This shift is within the astrometric accuracy of the star relative to Saturn.

Since the PSFs of Rhea and the star were nearly the same, we attempted to perform optimal stellar photometry, analogous to the optimal spectral extraction of Horne (1986) and others. One of us (J.H.) used this method for exoplanetary secondary eclipses from the Spitzer Space Telescope (Deming et al. 2005), but developed it initially for the present analysis. We divided each background-subtracted point-source image by a normalized PSF template. Each pixel in the subarray thus became an estimate of the total flux of the point source, with a variance that increased (rapidly) with distance from the stellar centroid. We then computed an error-weighted (i.e., optimal) mean of all the good pixels in the frame.

In principle, this process can reduce the noise by up to $70 \%$. The optimal weighting discounts pixels far from the stellar centroid, and thus reduces the effect of read noise and residual photon and scintillation noise from scattered planetary light. There is also no photometry aperture size decision to make. However, the method requires an accurate PSF model and good centroids. The highly varying and non-Gaussian PSFs of these short-exposure images precluded any smoothing or analytic PSF model fit, so the PSF templates were just the backgroundsubtracted, normalized images of Rhea, shifted to the centroided position of the star in its images and down-sampled to the original resolution. For both Rhea and the star, we used the background level from aperture photometry. 
Regrettably, while this procedure yielded a lower baseline that was three times less noisy than in the aperture-derived light curve, the upper baseline was about four times noisier. Close examination of the PSFs showed that noise and the fractional-pixel centroiding errors were sufficient to compromise this method. We decided, for the sake of consistency, not to merge the initial aperture photometry with the final optimal photometry. However, a much brighter PSF standard might have substantially improved our photometry. Obtaining such a standard may be difficult in occultation experiments with bright stars, but we note that large satellites could still play this role in occultations of fainter stars, potentially increasing the number of useful occultation events. If optimal photometry were successful, the disappearance of scintillation noise with atmospheric depth would argue for the application of significance tests similar to the scaled noise tests of Paper II, as opposed to the unscaled set.

We did not acquire useful egress data. Atmospheric emersion occurred in the rings (see Figure 1), so following Harrington et al. (1993) we employed a $3.34 \mu \mathrm{m}$ filter (wavelength measured warm) in which both planet and rings are dark due to overlapping absorption bands of water ice and methane. To increase the frame rate, NSFCAM's movieburst mode cannot display data during acquisition. It was thus not possible to see until afterward that the frames were empty. Possible reasons include a rise in the telluric water column (which would absorb all the light at this wavelength), a filter wheel problem, or (unlikely) a pointing problem. Two processes using shared memory on a dual-CPU camera computer could implement a movieburst mode with simultaneous image display without compromising the frame rate.

\section{TIMING AND ARRAY CLOCKING}

Relating each flux measurement to a location in the atmosphere requires precise knowledge of the time of each exposure. At the start of the first movieburst frame, NSFCAM's computer reads the time from a Global Positioning System (GPS) receiver. The observer must find the delay between the GPS read and the actual first collection of photons, the actual exposure time, and the time to read the array (which is done many times per frame). For this purpose, there is a flashing light-emitting diode (LED) that is driven by the GPS and located in the camera. The LED turns on for $0.1 \mathrm{~s}$ at the start of each Coordinated Universal Time (UTC) second. It is often lit for only part of an exposure, resulting in count levels that are between the fully lit and unlit levels. By observing this LED with exactly the same settings as for the occultation, one can model the count levels to determine the timing parameters.

We made such measurements before and after the occultation. For each frame, we summed the flux in the sky subarray (which was lit by the LED) after subtracting from each pixel the average per-pixel flux in the star subarray (which was not).

Our model starts with five lists of event times: frame resets, pedestal non-destructive reads (NDRs; see below), readout NDRs, LED “on" times, and LED "off" times. It concatenates the lists, sorts them in time, and steps through the result to calculate the flux per frame. It thus takes into account the changes in effective sensitivity induced by each NDR. The five free parameters are delay after GPS read, integration time, readout time, and the two LED flux levels. We iteratively fit the model to the LED data by minimizing the rms residuals. The relevant results appear in Figure 3 and Table 2. The electronic supplement to this article includes code that implements the

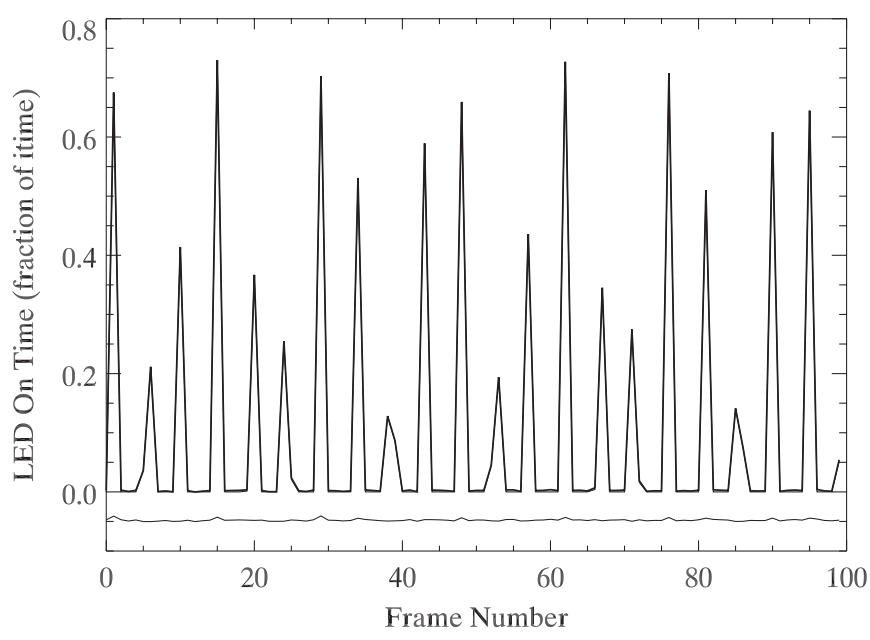

Figure 3. Portion of the LED timing light curve and the best-fit model. Traces for both the LED light curve and the model overplot each other at the top of the plot. The lowest trace presents the residuals, offset by -0.05 for clarity.

model in the Interactive Data Language (a product of ITT Visualization Systems, Boulder, CO, USA).

The per-image timing uncertainty ("jitter") is difficult to estimate, as fluctuations in the LED brightness, read noise, etc. all masquerade as jitter. This parameter is important in studies of detailed light curve structure, such as the shape and location of spikes, but this is not the focus of our present investigation.

Future instruments will likely read the GPS for each frame. However, an end-to-end test such as that provided by NSFCAM's LED builds confidence that these increasingly sophisticated instruments have consistent timing. It also quantifies the actual integration time and the delay between the GPS read and the first detected photons.

We used NSFCAM's default "array reset clocking, double sample" mode. This mode performs a series of NDRs both before and after the exposure and records the difference between the two sets' averages. The multiple NDRs suppress read noise but reduce sensitivity for part of the exposure. We used the NSFCAM default of eight NDRs, but should have reduced dead time and increased read noise by using fewer NDRs. There were two effects of our long dead time. First, the photon noise was higher than it could have been. Since scintillation still dominated the noise even after our correction, this is regrettable but not serious. Second, flux variations occurring during either set of NDRs were only fractionally recorded, depending on when they occurred in the set. We may have missed some of the light in fast spikes. These correspond to the smallest-scale features in the inversion presented in Paper II. Since the small scales have the smallest amplitudes (as expected, see Wavelet Analysis, Paper II), we conclude that our error did not strongly affect the results.

\section{CONCLUSIONS}

We have presented a light curve based on IRTF observations of the 1998 November 14 occultation of GSC 0622-00345 by Saturn. The light curve has a per-frame $S / N$ of 66 and a perscale-height $\mathrm{S} / \mathrm{N}$ of 267 , placing it among the best ground-based atmospheric light curves. We have described the observational and analytic methods used to derive the light curve, in some cases for the first time in the literature, although these techniques are known among experienced occultation observers. Methods that improved the photometry include using Rhea as a scintillation and pointing standard, spatially subsampling the data by 
a factor of 10 for accurate aperture placement, and removing a template of scattered light. Separate, identical, measurements of a flashing LED placed in the telescope beam provided an independent measure of the frame rate, when fit by a detailed model of the chip's sensitivity throughout the imaging cycle. Optimal photometry shows promise at reducing the photometric noise level as the star dims, but requires a PSF standard that is substantially brighter than the occultation star. Occultation cameras with wider fields of view (but still able to operate with accurate timing at high frame rates) would facilitate finding bright PSF standards.

We thank P. Nicholson for his pre-occultation calculations and J. L. Elliot, E. Lellouch, and L. Young for comments that significantly improved the paper. We thank the NASA Astrophysics Data System, JPL Solar System Dynamics group, and the free and open-source software communities for software and services. This investigation was supported by Wellesley
College under NASA Contract 961169 and by the NASA PGG program.

Facilities: IRTF

\section{REFERENCES}

Bosh, A. S., \& McDonald, S. W. 1992, AJ, 103, 983

Deming, D., Seager, S., Richardson, L. J., \& Harrington, J. 2005, Nature, 434, 740

French, R. G., Elliot, J. L., \& Gierasch, P. J. 1978, Icarus, 33, 186

Glass, I. S. 1999, Handbook of Infrared Astronomy (Cambridge: Cambridge Univ. Press)

Harrington, J., Cooke, M. L., Forrest, W. J., Pipher, J. L., Dunham, E. W., \& Elliot, J. L. 1993, Icarus, 103, 235

Harrington, J., French, R. G., \& Matcheva, K. 2010, ApJ, 716, 404 (Paper II)

Høg, E., et al. 2000, A\&A, 355, L27

Horne, K. 1986, PASP, 98, 609

Hubbard, W. B., et al. 1997, Icarus, 130, 404

Lang, K. R. 1992, Astrophysical Data. I. Planets and Stars (Berlin: Springer)

Shure, M., et al. 1994, Exp. Astron., 3, 239

Skrutskie, M. F., et al. 2006, AJ, 131, 1163 